\title{
RNAi-Mediated Silencing of the Chitinase 5 Gene for Fall Webworm (Hyphantria cunea) Can Inhibit Larval Molting Depending on the Timing of dsRNA Injection
}

\author{
Xun Zhang, Yue Wang, Sufang Zhang, Xiangbo Kong, Fu Liu and Zhen Zhang *
}

check for updates

Citation: Zhang, X.; Wang, Y.; Zhang, S.; Kong, X.; Liu, F.; Zhang, Z. RNAi-Mediated Silencing of the Chitinase 5 Gene for Fall Webworm (Hyphantria cunea) Can Inhibit Larval Molting Depending on the Timing of dsRNA Injection. Insects 2021, 12, 406. https://doi.org/10.3390/ insects12050406

Academic Editor: Steve Whyard

Received: 1 March 2021

Accepted: 29 April 2021

Published: 30 April 2021

Publisher's Note: MDPI stays neutral with regard to jurisdictional claims in published maps and institutional affiliations.

Copyright: (C) 2021 by the authors Licensee MDPI, Basel, Switzerland. This article is an open access article distributed under the terms and conditions of the Creative Commons Attribution (CC BY) license (https:// creativecommons.org/licenses/by/ $4.0 /)$.
Key Laboratory of Forest Protection of National Forestry and Grassland Administration, Research Institute of Forest Ecology, Environment and Protection, Chinese Academy of Forestry, Beijing 100091, China; zhx3355@126.com (X.Z.); cafwy1016@163.com (Y.W.); Zhangsf@caf.ac.cn (S.Z.); xbkong@sina.com (X.K.); liufu@sina.com (F.L.)

* Correspondence: zhangzhen@caf.ac.cn

Simple Summary: The fall webworm, Hyphantria cunea, is a worldwide invasive pest causing serious ecological and economic damage. The use of RNAi is a feasible strategy for controlling this pest. In this study, we evaluated the importance of the chitinase 5 gene ( $\mathrm{HcCht5}$ ) in the development of H. cunea larvae. We found that the RNAi-mediated silencing of HcCht5 arrested molting and caused larval mortality depending on the dsRNA injection time. The silencing of HcCht5 downregulated genes that were related to chitin metabolism, molting hormone signaling, and detoxification metabolism. Our findings indicate that $\mathrm{HcCht5}$ is an important gene in regulating larval development and a promising target for RNAi-mediated pest management of the pest $H$. cunea.

Abstract: Chitinases, which are crucial enzymes required for chitin degradation and reconstruction, are often selectively considered to be effective molecular targets for pest control due to their critical roles in insect development. Although the Hyphantria cunea chitinase gene has been reported previously, its sequence characteristics, gene function, and feasibility as a potential target for pest management were absent. In the present study, we characterized the H. cunea chitinase gene and designated it $\mathrm{HcCht5}$. Phylogenic and domain structure analysis suggested that $\mathrm{HcCht5}$ contained the typical chitinase features and was clustered into chitinase group I. Tissue-specific and developmental expression pattern analysis with Real-Time Quantitative PCR (RT-qPCR) showed that $\mathrm{HcCht5}$ was mainly expressed in the integument tissues and that the transcript levels peaked during molting. RNA interference (RNAi)-mediated silencing of $\mathrm{HcCht5}$ caused $33.3 \%$ (2 ug) and $66.7 \%$ (4 ug) mortality rates after double-stranded RNA (dsRNA) injection. Importantly, the interference efficiency of $\mathrm{HcCh} 5 \mathrm{f}$ depended on the injection time of double-stranded RNA (dsRNA), as the premolting treatment achieved molt arrest more effectively. In addition, transcriptome sequencing (RNA-seq) analysis of RNAi samples demonstrated silencing of the down-regulated HcCht5 genes related to chitin metabolism and molting hormone signaling, as well as genes related to detoxification metabolism. Our results indicate the essential role of HcCht5 in H. cunea development and detail the involvement of its gene function in the larval molting process.

Keywords: Hyphantria cunea; chitinase; molting; RNAi efficiency; transcriptome analysis

\section{Introduction}

Chitin, a linear polymer made of $N$-acetyl- $\beta$-D-glucosamine (GlcNAc) linked by $\beta-1,4$ glycosidic bonds, is an important component of the epidermis and peritrophic matrix in insects [1]. The balance of the chitin content is particularly essential for insect development (molting) and metamorphosis. Chitin synthesis and degradation occur simultaneously during insect molting, when the old cuticle is degraded and replaced with the new cuticle formed by the underlying epidermal cells [2]. Multiple enzymes are involved in this process 
of chitin metabolism. Chitinases (Chts, E.C.3.2.1.14), which belong to glycoside hydrolase family 18 (GH18), are key enzymes in the degradation of chitin. The function of chitinase is to hydrolyze the linear polymer of chitin and degrade chitin into low-molecular-weight chitooligosaccharides. Insect chitinases are essential for insect survival and development. They appear to play roles in cuticle turnover, wing expansion, digestion, immunity and natural defense [1,3]. Insect chitinase was first cloned from Manduca sexta [4]. Since then, hundreds of chitinase genes have been continuously reported from different insect species, such as Tribolium castaneum [3], Spodoptera litura [5], Choristoneura fumiferana [6], Helicoverpa armigera [7], Spodoptera exigua [8], Locusta migratoria [9], and Sogatella furcifera [10].

Currently, chitinases and chitinase-like proteins are classified into 11 groups (groups I to $X$, and the Lepidoptera-specific group $h$ ) based on their sequence similarities, domain architectures, tissue-specificity of expression, and functions [11-13]. Among them, group I $(C h t 5)$ has been relatively well characterized. The transcripts of $C h t 5 s$ are mainly detected in the epidermis and the gut [7], which suggests that Cht5s may be involved in chitin turnover in the cuticular exoskeleton and peritrophic membrane. Heterologous expression of Cht5 of Drosophila melanogaster and T. castaneum has also been successfully performed in the Hi-5 cell lines, and the recombinant protein showed high levels of chitinolytic activity [13]. At present, most studies on Cht5 revealed its function in pupal-adult or nymph-adult molting in coleopteran, orthopteran, and homopteran insects using RNA interference (RNAi) technology. For instance, in T. castaneum, TcCht5 was down-regulated by the injection of TcCht5-specific double-stranded RNA (dsRNA) into larvae, which led to pupal-adult molting arrest [3]. In L. migratoria, two Cht5 genes, LcCht5-1 and LcCht5-2, were identified. The RNAi of LcCht5-1 disrupted molting from nymphs to adults [14]. In Sogatella furcifera, silencing of $S f C h t 5$ also affected the nymph-adult transition [10]. However, the function of Cht5 in larvae-larvae molting in Lepidoptera has been rarely studied.

The fall webworm, Hyphantria cunea Drury, is a Lepidoptera insect belonging to the family of Arctiidae. H. cunea is a worldwide invasive pest, and the larval stage is the main stage in which the larvae can attack and feed on more than 600 host plants, causing serious ecological and economic damage [15]. Currently, monitoring and control of H. cunea is primarily achieved through chemical insecticides, which are environmentally unfriendly. Some parasitoid wasps, sex pheromone traps, and biopesticides are also used, but are inefficient and costly [16-19]. The identification of novel management strategies is urgently required to control this pest. In recent years, RNAi-based pest management has been increasingly studied as a novel insect control strategy and the publication of genome and transcriptome sequencing for H. cunea also provided a molecular basis for H. cunea control [20-22]. However, potentially effective genetic targets and innovative strategies to control this invasive pest are still lacking. Chitinase 5 genes from different insects have been identified as potential silencing targets and resulted in abnormal molting and increased mortality in the pupal-adult or nymph-adult process [23,24]. However, for H. cunea, the larval stage is the important damage stage and the key period for its control. Little research has focused on chitinase genes from H. cunea. Although a chitinase gene (Cht5, Accession number: U86877) was cloned by Kim et al. in H. cunea in 1998 [25], its sequence characteristics, gene function, and feasibility as a potential target for pest control were absent. To research the function of this chitinase gene in the H. cunea larvae molting process, we further identified and analyzed the function of this gene using the RNAi method and considered the possibility of its use as one of the competitive molecular targets for H. cunea control.

In the present study, we identified and analyzed the domain structure of $H$. cunea chitinase gene ( $\mathrm{HcCht5)}$, a group I gene, and profiled its tissue-specific and developmental expression patterns. RNAi and transcriptome sequencing (RNA-seq) were used to gain insights into the biological function of HcCht5. Our results revealed the crucially important role of $\mathrm{HcCht5}$ during larval molting and in the promotion of the development of RNAibased management of H. cunea control. 


\section{Materials and Methods}

\subsection{Insect Rearing}

H. cunea larvae were kindly provided by the laboratory of Insect Virus Research Center, Chinese Academy of Forestry. They were reared on an artificial diet under a $14 \mathrm{~h}$ light: $10 \mathrm{~h}$ dark photoperiod at $26( \pm 1){ }^{\circ} \mathrm{C}$ with $75 \%( \pm 10 \%)$ relative humidity.

\subsection{Cloning and Sequencing of the HcCht5 cDNA}

The total RNA was isolated from the third instar larvae on the second day after molting using the Trizol Plus reagent (Ambion, Austin, TX, USA), following the manufacturer's recommended protocol. The RNA concentration and quality were assessed using a spectrophotometer (Denovix, Wilmington, DE, USA) and 1\% agarose gel electrophoresis. cDNA synthesis was performed by the GoScript ${ }^{\mathrm{TM}}$ Reverse Transcription System kit (Promega, Madison, WI, USA) with an oligo $(\mathrm{dT})_{15}$ primer, and 1 ug of total RNA was used per reaction.

The full-length coding sequence of $\mathrm{HcCh}+5$ was amplified using PrimeSTAR ${ }^{\circledR}$ Max DNA Polymerase (Takara, Shanghai, China). PCR primers were designed based on the reported H. cunea chitinase gene (Accession number: U86877) with the Primer Premier 5 software. The primer sequences are listed in Table S1. The PCR program used was as follows: $94{ }^{\circ} \mathrm{C}$ for $3 \mathrm{~min}$; 35 cycles of $94{ }^{\circ} \mathrm{C}$ for $30 \mathrm{~s}, 55^{\circ} \mathrm{C}$ for $30 \mathrm{~s}$ and $72{ }^{\circ} \mathrm{C}$ for $2 \mathrm{~min}$; and $72{ }^{\circ} \mathrm{C}$ for $10 \mathrm{~min}$. The PCR products were purified and cloned into a pEASY-Blunt 3 vector (TransGen, Beijing, China) and sequenced at Sangon Biotech (Co., Ltd., Beijing, China).

\subsection{Deduced Amino Acid Sequence Analysis of HcCht5}

The amino acid sequences were deduced using the Translate tool on the ExPASy Proteomics website (http:/ / web.expasy.org/translate/) (accessed on 20 February 2020). The molecular weight and isoelectric point $(p I)$ of HcCht5 were predicted using the Compute pI/Mw tool (https:/ / web.expasy.org/compute_pi/) (accessed on 20 February 2020). SMART domain analysis (http:/ / smart.embl-heidelberg.de/) (accessed on 20 February 2020) and SignalP 4.1 Server (http:/ / www.cbs.dtu.dk/services/SignalP/) (accessed on 20 February 2020) were used to predict the domain architecture and signal peptide. Percent identity analysis of HcCht5 was conducted by NCBI blastp (https:/ / blast.ncbi.nlm.nih. gov / Blast.cgi) (accessed on 25 February 2020). To compare the amino acid sequences and catalytic domains of HcCht5 along with other insect orders, multiple amino acid sequence alignment was carried out using Clustal X software [26], and the identities among Chts were analyzed by GeneDoc software (http: / / wwwpscedu/biomed/genedoc) (accessed on 25 February 2020). Four conserved motifs were identified based on the references previously described by Arakane and Muthukrishnan [1] and Zhang et al. [27]. The known insect Cht5 genes in other insect orders deposited in GenBank were used to construct phylogenetic trees using the MEGA 6.0 software package with the neighbor-joining method. Bootstrap analysis was performed, and the robustness of each cluster was confirmed in 1000 replications.

\subsection{Tissue-Specific and Developmental Expression Analysis of HcCht5}

For tissue-specific expression analysis, different tissues (head, integument, gut, and fat bodies) were dissected from larvae on the second day of the fifth instar stadium (L5D2). The larvae were first kept on ice for $3 \mathrm{~min}$ and then dissected with sterile insect scalpels under a zoom stereomicroscope (Olympus, SZX7). The heads from ten larvae and other tissues (integument, gut, and fat bodies) from three larvae were pooled as one treatment. Each treatment contained three biological replicates. For developmental expression analysis, the whole larva was sampled and used for developmental expression analysis. The first to fifth instar larvae (L1 to L5) larvae on the third day of the stadium (D3) and the fourth instar larvae from day 1 to day 5 of the stadium (L4D1 to L4D5) were collected. Samples from each developmental stage contained three biological replicates. At least $100 \mathrm{mg}$ of tissue 
or larvae were sampled per replicate. All collected samples were immediately frozen in liquid nitrogen and stored at $-80^{\circ} \mathrm{C}$.

\section{5. $R T-q P C R$}

To screen for the stable reference genes of H. cunea, qRT-PCR was employed to analyze the expression of four candidate reference genes ( $\beta$-actin, $\beta$-tubulin, GAPDH, and $E F 1 \alpha$ ) at different developmental stages (L1 to L5) and different tissues (head, integument, gut, and fat bodies), respectively. RT-qPCR was performed using the SuperReal PreMix Plus (SYBR Green) kit (TIAN GEN, Co., Ltd., Beijing, China) with a $20 \mu \mathrm{L}$ reaction. Each reaction contained the following: $10 \mu \mathrm{L} 2 \times$ SuperReal PreMix Plus (SYBR Green) solution, $0.6 \mu \mathrm{L}$ forward and reverse primers in a final concentration of $10 \mu \mathrm{M}, 7.8 \mu \mathrm{L}$ nuclease free water, and $1 \mu \mathrm{L}$ of undiluted cDNA. RT-qPCR was carried out using a LightCycler $480 \mathrm{II}$ (Roche, Basel, Switzerland) with the following conditions: $95^{\circ} \mathrm{C}$ for $3 \mathrm{~min}$, followed by 45 cycles at $95^{\circ} \mathrm{C}$ for $5 \mathrm{~s}$, and $60^{\circ} \mathrm{C}$ for $30 \mathrm{~s}$. Each reaction included three technical repetitions. According to the analysis by NormFinder (https: / wwww.moma.dk/normfindersoftware/) (accessed on 15 March 2020), the stabilities of the four candidate reference genes were analyzed.

All RT-qPCR experiments were conducted with the methods above. Each treatment included three biological replicates and three technical repetitions. The relative mRNA levels of the target genes were calculated using the $2^{-\Delta \Delta C t}$ method by normalizing them to the expression of the screened reference gene. All the PCR primers were designed using the Primer Premier 5 software. The primer sequences are listed in Supplementary Table S1. Melting curve analyses were performed for all the primers.

\section{6. dsRNA Synthesis and RNAi of HcCht5}

The dsRNA was synthesized using a T7 RiboMAX ${ }^{\mathrm{TM}}$ Express RNAi System (Promega, Madison, WI, USA) in accordance with the manufacturer's instructions. T7 promoter sequences were tailed to the $5^{\prime}$-ends of the DNA templates by PCR amplification. The primer sequences are listed in Supplementary Table S1. Template DNA and single-stranded RNA were removed from the transcription reaction by DNase and RNase treatments, respectively.

For RNAi, larvae from the last day of the third stadium (L3D5) with similar sizes and growth conditions were selected, 2 ug and 4 ug of dsHcCht 5 or dsGFP (control) solution were injected into each larva through the abdominal side between the fourth and fifth abdominal segments, respectively, using a microinjector (Hamilton, Bonaduz, Switzerland). A total of 12 larvae were treated for each group, three larvae (replicates) were randomly sampled from each treatment group at $12 \mathrm{~h}$ and $24 \mathrm{~h}$ post treatment, immediately frozen in liquid nitrogen, and stored at $-80^{\circ} \mathrm{C}$. RNA extraction and RT-qPCR detection of the relative transcript level of $\mathrm{HcCht5}$ were conducted as described above. For RNAi phenotype observation, another 30 larvae were treated for each group, the abnormal rate was investigated at the molting stage, and the mortality rate was investigated five days post treatment.

To research the RNAi efficiency of $\mathrm{HcCh}$ t5 at different injection times, 30 larvae were separately injected with 4 ug dsHcCht5 from day 1 to day 5 of the fourth instar larvae stadium (L4D1 to L4D5). The control group was injected with the same amount of dsGFP. The abnormal rate and mortality rate were investigated at the molting stage and five days post molting, respectively.

\subsection{RNA-Seq and Analysis}

At the first day of the fourth stadium, the larvae were injected with 2 ug dsHcCht5 or dsGFP (control). Three larvae (biological replicates) from each treatment were collected $12 \mathrm{~h}$ post injection, immediately frozen in liquid nitrogen, and then stored at $-80^{\circ} \mathrm{C}$. For mRNA sequencing, the total RNA was extracted as described above. RNA degradation and contamination were monitored on $1 \%$ agarose gels. RNA purity was checked using the NanoPhotometer ${ }^{\circledR}$ spectrophotometer (IMPLEN, Westlake Village, CA, USA). The RNA 
concentration was measured using a Qubit ${ }^{\circledR}$ RNA Assay Kit in Qubit ${ }^{\circledR}$ 2.0 Flurometer (Life Technologies, Carlsbad, CA, USA). The RNA integrity was assessed using the RNA Nano 6000 Assay Kit of the Agilent Bioanalyzer 2100 system (Agilent Technologies, Santa Clara, CA, USA).

A total amount of $3 \mu \mathrm{g}$ RNA per sample was used as input material for the RNA sample preparations. Sequencing libraries were generated using NEBNext ${ }^{\circledR}$ Ultra $^{\mathrm{TM}}$ RNA Library Prep Kit for Illumina ${ }^{\circledR}$ (NEB, Ipswich MA, USA) following the manufacturer's recommendations, and index codes were added to attribute sequences to each sample. The library preparations were sequenced on an Illumina HiSeq2000 platform (Illumina, San Diego, CA, USA) by Biomarker Technologies (Co., Ltd., Beijing, China).

The raw data outputs from the Illumina equipment were trimmed for adapters, and polyA/T tails and low-quality reads (Q20 less than 20) were removed to obtain high-quality clean reads. The clean reads were assembled to produce unigenes using Trinity [28]. The assembled unigenes were aligned with the NR databases using BLAST (http:/ /blast.ncbi. nlm.nih.gov/Blast.cgi) (accessed on 10 August 2020) with a cut-off E-value of $10^{-5}$.

The unigene abundance was measured as the fragments per kilobase of transcript per million mapped reads (FPKM) using RSEM [29]. The identification and counting of differentially expressed genes (DEGs) between dsGFP- and dsHcCht5-treated samples were conducted with the DESeq R package (1.10.1) at a False Discovery Rate (FDR) of $\leq 0.05$ and a $\log _{2}$ fold change of $\geq 1.5$, followed by hierarchical clustering based on the expression values. DEGs were validated by RT-qPCR as described above. DEG analysis was performed using BMKCloud (www.biocloud.net) (accessed on 10 September 2020).

\subsection{Statistical Analysis}

For the analysis of $\mathrm{HcCh}$ t5 expression patterns in different tissues and at different developmental stages, one-way analysis of variance followed by Tukey's test was applied. The other data were analyzed statistically using an independent sample Student's $t$-test. In the figures, different letters above the bars represent significant differences in the HcCht5 expression between the samples $(p<0.05)$, while asterisks are used to indicate significant differences $\left({ }^{*}, p<0.05\right)$.

\section{Results}

\subsection{Sequence Analysis of $\mathrm{HcCh}+5$}

The full-length coding sequence of the HcCht5 gene had a $1662 \mathrm{bp}$ ORF encoding 553 amino acid residues with a predicted molecular weight of $61.97 \mathrm{kDa}$ and a predicted pI of 4.96. The deduced amino acid sequence of HcCht5 showed $80.07 \%$ identity with BmCht5. The domain architecture of $\mathrm{HcCht} 5$ contained a signal peptide (amino acids 1-20), a catalytic domain (GH18 domain, amino acids 24-376), and a chitin-binding domain (amino acids 496-553) (Figure 1A). Catalytic motif analysis showed that HcCht5 possesses four conserved catalytic domains: KXXXAVGGW, FDGXDLDWEYP, MXYDLRG, and GAMXWAIDMDD, where $X$ is a nonspecific amino acid (Figure 1B). These motifs are considered the catalytic active sites of chitinase. Phylogenetic analysis showed that HcCht5 exhibited a high homology with BmCht5 and was clustered in the branch of group I chitinases (Figure S1).

\subsection{Tissue-Specific and Developmental Expression of HcCht5}

RT-qPCR was used to analyze the spatiotemporal expression levels of HcCht5. To screen for stable reference genes of $H$. cunea, four candidate reference genes ( $\beta$-actin, $\beta$-tubulin, $G A P D H$, and $E F 1 \alpha$ ) were evaluated for their expression stability in different developmental stages and different tissues. Finally, $\beta$-Actin showed the most stability and was used as the reference gene for RT-qPCR analysis (Table S3). To investigate the tissue-specific expression of $\mathrm{HcCht5}$, different tissues (head, integument, gut, and fat bodies) from the fifth instar larvae were dissected and tested. The results showed that $\mathrm{HcCht5}$ was expressed in all the tested tissues and most highly expressed in the integument (Figure 2A). 
A

HcCht5
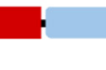

B

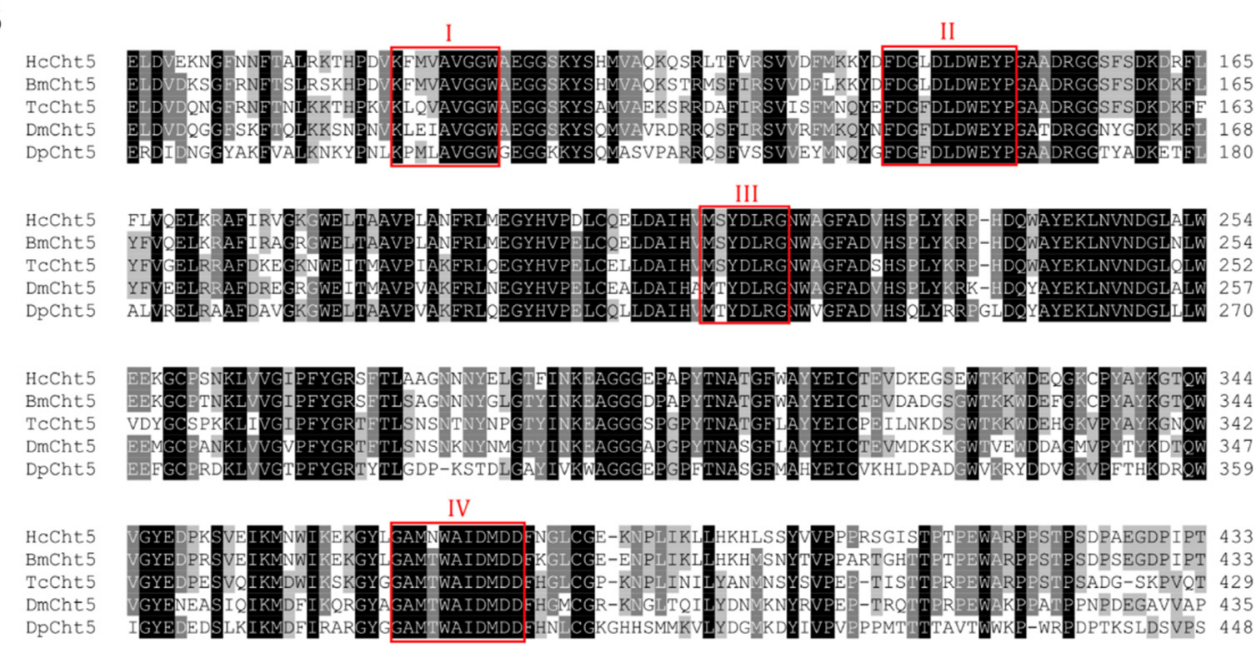

Figure 1. Domain architectures and catalytic motif analysis of H. cunea chitinase (HcCht5). (A) Domain architectures of HcCht5. (B) Conserved domains in the glycoside hydrolase family 18 (GH18) of HcCht5 and four other group I chitinases (BmCht5, TcCht5, DmCht5, and DpCht5). The conserved and similar amino acid residues are labeled with black (100\% identity) and gray (60-80\% identity) backgrounds, respectively. Red-boxed regions are the four conserved motifs (I-IV) represented by the sequences KXXXAVGGW, FDGXDLDWEYP, MXYDLRG, and GAMXWAIDMDD, where $X$ is a nonspecific amino acid. Four insect chitinases belonging to chitinase group I (BmCht5, TcCht5, DmCht5, and DpCht5) were used for catalytic motif analysis. Their GenBank accession numbers are listed in Table S2.

A

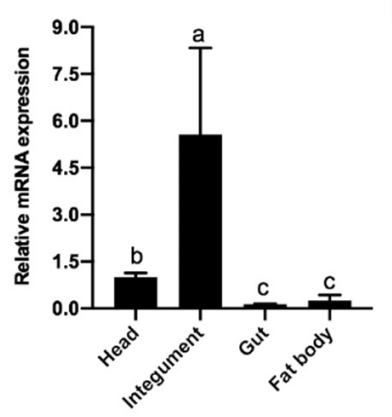

B

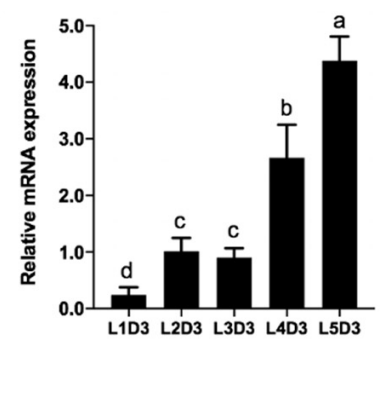

C

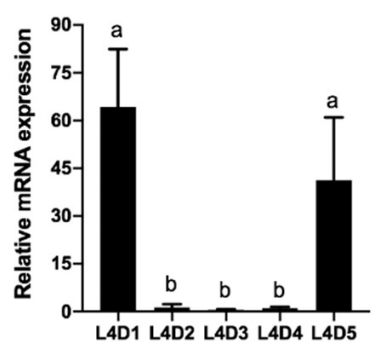

Figure 2. The expression of HcCht5 in H. cunea by RT-qPCR. (A) The expression of HcCht5 in different tissues of day 3 of the fifth instar larvae. (B) The expression of HcCht5 on the third day of each larval stadium (L1D3-L5D3). (C) The expression of HcCht5 on each day of the fourth larval stadium (L4D1L4D5). Error bars represent the standard error of the calculated means based on three biological replicates. Different letters above the bars represent significant differences in the HcCht5 expression between the samples (one-way analysis of variance followed by Tukey's test, $p<0.05$ ).

To investigate the developmental expression of $\mathrm{HcCht5}$, we detected the expression level of HcCht5 in day 3 larvae from the first instar to the fifth instar (L1D3-L5D3) and in larvae from the first day to the last day of the fourth instar (L4D1-L4D5), respectively. The results showed that HcCht5 was expressed at a low level in the early stage (L1 to L3) and a high level at the fourth and the fifth instar (L4 and L5) (Figure 2B). The HcCht5 
mRNA displayed an extremely high expression level at L4D1 and L4D5, but an extremely low expression at L4D2 to L4D4 (Figure 2C). This indicates that HcCht5 was significantly up regulated during molting and rapidly disappeared during inter-molting, implying a conceivable role of $\mathrm{HcCht5}$ during the molting process.

\subsection{RNAi of HcCht5}

To investigate the effect of HcCht5 on H. cunea molting, RNAi was performed by the injection of dsHcCht5 and dsGFP (control). Since HcCht5 was highly expressed pre-molting, we selected larvae on the fifth day of the third instar (the day before molting) for RNAi. RTqPCR was carried out to test the transcription level of HcCht5 after treatment. The results showed that the expression of $\mathrm{HcCht5}$ was effectively reduced at $12 \mathrm{~h}$ but recovered at $24 \mathrm{~h}$ after the injection of 2 ug dsHcCht5 against dsGFP (Figure 3A). In contrast, a concentration of $4 \mathrm{ug}$ dsHcCht5 resulted in significant silencing at both $12 \mathrm{~h}$ and $24 \mathrm{~h}$ post injection (Figure 3B). In addition, phenotypic observations suggested that all the larvae in the control group could molt normally, whereas the dsHcCht5 injection group showed $33.3 \%$ ( $2 \mathrm{ug}$ ) and $66.7 \%$ (4 ug) abnormal molting (Table 1). These abnormalities were characterized by the fact that some larvae could shed the epidermis but did not molt completely, some larvae stopped molting halfway, and some could not molt at all (Figure 3C). Finally, the dsHcCht5 injection resulted in 33.3\% (2 ug) and 66.7\% (4 ug) mortality rates at five days post treatment, while no death was observed in the control group (Table 1). These results demonstrated the crucially important role of HcCht5 during H. cunea larval molting.

A

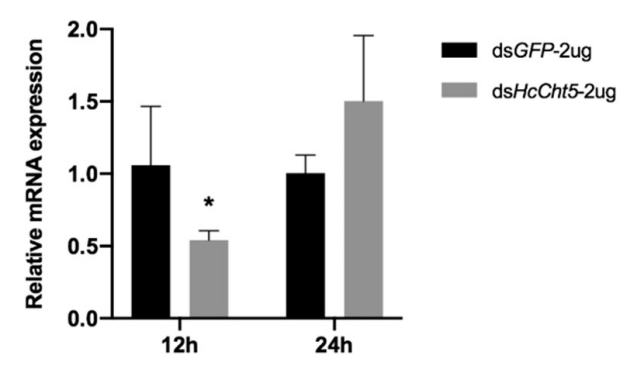

B

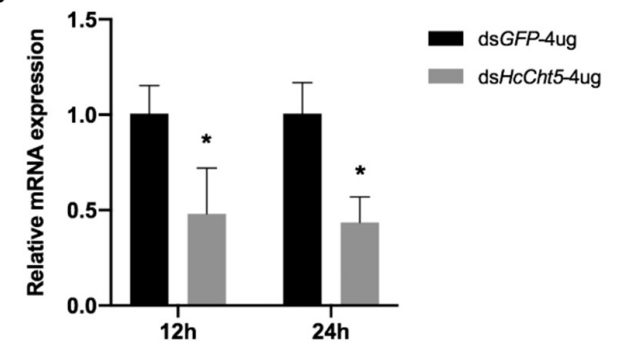

C
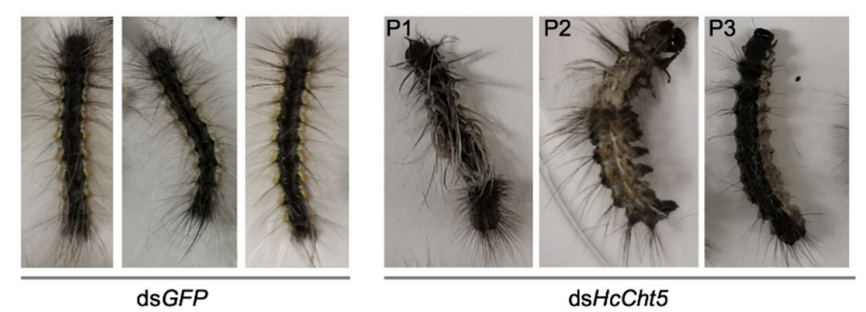

Figure 3. RNAi efficiency of HcCht5. (A) The relative mRNA expression levels of $H c C h t 5$ after injection with $2 \mathrm{ug}$ dsHcCht5 and dsGFP. (B) The relative mRNA expression levels of $\mathrm{HcCht5}$ after injection with $4 \mathrm{ug}$ dsHcCht5 and dsGFP. dsRNAs were injected in L3D5 instar (the day before molting) larvae. The relative mRNA expression levels of $\mathrm{HcCh} t 5$ were determined using RT-qPCR at $12 \mathrm{~h}$ and $24 \mathrm{~h}$ post injections. Error bars represent the standard error of the calculated means based on three biological replicates. Asterisks indicate significant differences (Student's $t$-test, $p<0.05$ ). (C) The abnormal molting phenotypes of $\mathrm{H}$. cunea larvae after injection with dsHcCht5. Insects injected with dsGFP were able to molt successfully, while the dsHcCht5-injected insects displayed three abnormal molting phenotypes: P1, some larvae could shed the epidermis but did not molt completely; P2, some larvae stopped molting halfway; and P3, some larvae completely failed to molt.

Considering the expression pattern of $\mathrm{HcCht5}$, which displayed an extremely high expression during ecdysis and then followed a sharp decline after ecdysis (Figure 2C), we wondered whether the injection time could affect the RNAi efficiency. Therefore, injections of dsRNA at different stages of the fourth instar larvae (L4D1-L4D5) were conducted. We 
found that only the treatment at L4D4 and L4D5 resulted in the molting defect and lethal phenotype, whereas no phenotypic changes were detected for treatment at L4D1, L4D2 and L4D3 (Table 2). In addition, compared with the dsGFP control, injection of dsHcCht5 at L4D5 resulted in a higher abnormal rate $(26.7 \%)$ and mortality rate $(26.7 \%)$ than the injection at L4D4, which showed a 10\% abnormal rate and 10\% mortality rate, respectively (Table 2). These results indicated that the injection time of dsHcCht5 could affect the RNAi efficiency of $\mathrm{HcCht5.}$

Table 1. The abnormal rate and mortality rate of $H$. cunea after injection of dsHcCht5 in different concentrations.

\begin{tabular}{ccccc}
\hline \multirow{2}{*}{ Treatment $^{1}$} & dsGFP & dsHcCht5 & dsGFP & dsHcCht5 \\
\cline { 2 - 5 } & \multicolumn{2}{c}{ 2 ug/Larva $^{2}$} & \multicolumn{2}{c}{$\mathbf{4}$ ug/Larva } \\
\hline Abnormal rate & ND & $33.3 \%$ & ND & $66.7 \%$ \\
Mortality rate & ND & $33.3 \%$ & ND & $66.7 \%$ \\
\hline
\end{tabular}

${ }^{1}$ A total of 30 insects on the fifth day of the third instar were injected for each treatment group. The abnormal rate was investigated at molting stage of the third instar larvae. The mortality rate was investigated five days after treatment. dsGFP, the control group; dsHcCht5, the treatment group; and ND, not detected.

Table 2. The abnormal rate and mortality rate of $\mathrm{H}$. cunea after the injection of $\mathrm{ds} \mathrm{HcCht} 5$ at each day of the fourth larval stadium (L4D1 to L4D5).

\begin{tabular}{cccc}
\hline Treatment $^{\mathbf{1}}$ & & dsGFP & dsHcCht5 \\
\hline Abnormal rate & L4D1 & ND & ND \\
& L4D2 & ND & ND \\
& L4D3 & ND & ND \\
& L4D4 & ND & $10.0 \%$ \\
L4D5 & ND & $26.7 \%$ \\
\hline Mortality rate & L4D1 & ND & ND \\
& L4D2 & ND & ND \\
& L4D3 & ND & $10.0 \%$ \\
& L4D4 & ND & $26.7 \%$ \\
\hline
\end{tabular}

${ }^{1}$ A total of 30 insects were injected with 4 ug dsGFP/dsHcCht5 at each day of the fourth larval stadium (L4D1 to L4D5). The abnormal rate was investigated at the molting stage of the fourth larval stadium. The mortality rate was investigated five days post molting. dsGFP, the control group; dsHcCht5, the treatment group; and ND, not detected.

\subsection{Differentially Expressed Gene (DEG) Analysis after HcCht5 RNAi}

To investigate the effect of $\mathrm{HcCh}+5 \mathrm{RNAi}$ on other genes, RNA-seq analysis was used to identify genes that were differentially expressed after HcCht5 RNAi. A total of 154,927,972 clean reads were yielded after filtering out the adapter sequences and low-quality reads (Table S4). The gene expression profile of biological replicates from the same group showed a high correlation coefficient (Figure S2). According to the results of the transcriptome assembly, 54,852 unigenes were obtained and annotated to the NR database. Compared with dsGFP, 65 unigenes were identified as being differentially expressed in dsHcCht5 $(\log 2 \mathrm{FC}>1.5$, FDR $<0.05)$, among which $77 \%$ were down-regulated and $23 \%$ were up regulated (Figure 4A, Table S5). The heat map shows that the expression levels of DEGs were significantly different between the dsHcCht5 and dsGFP samples (Figure 4C). To confirm the differential gene expression, ten DEGs were selected for RT-qPCR validation. Most of the selected unigenes exhibited the same expression patterns as those observed in the transcriptome data (Figure S3). 
A

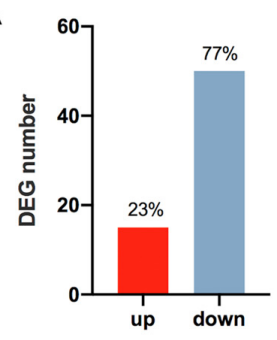

B

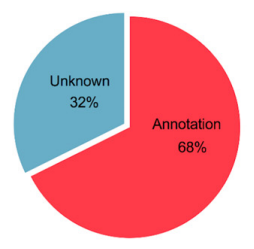

C

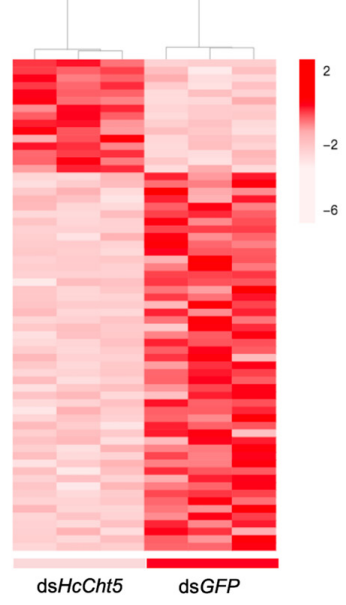

D

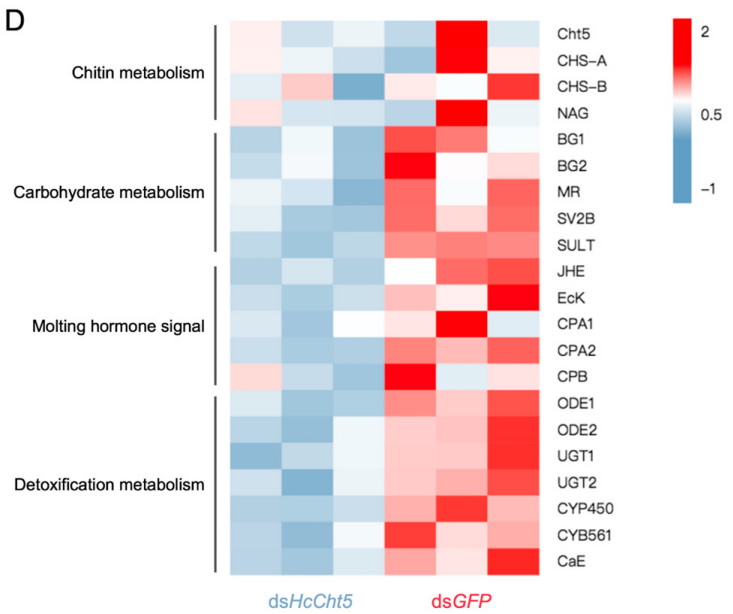

Figure 4. Differentially expressed gene (DEG) analysis of the dsHcCht5- and dsGFP-treated groups. (A) The number of DEGs in the dsHcCht5-treated group compared with the dsGFP group. Up indicates up regulated DEGs, and down indicates down-regulated DEGs. (B) The proportion of annotation and unknown DEGs. (C) Expression profiles of all DEGs between the dsHcCht5 and dsGFP groups. Each group contained three repeated samples. The color scale indicates the different expression levels, which were normalized as $\log _{10}$ fragments per kilobase of transcript per million mapped reads (FPKM) values. (D) Expression profiles of some of the most down-regulated DEGs and other interesting DEGs in the ds $\mathrm{HcCht5}$ and dsGFP groups. Each group contained three repeated samples. The color scale indicates the different expression levels. The letters on the right indicate putative gene names in H. cunea. Abbreviations: Cht5, chitinase 5; CHS-A and CHS-B, chitin synthase A and B; NAG, $\beta$-N-acetylglucosaminidase; BG1 and 2, $\beta$-glucosidase precursor 1 and 2; MR, putative mandelate racemase; SY2B, synaptic vesicle glycoprotein 2B-like; SULT, sulfotransferase; JHE, juvenile hormone esterase; EcK, ecdysteroid kinase; CPA and B, carboxypeptidase A and B; ODE1 and 2, odorant-degrading enzymes; UGT 1 and 2, UDP-glycosyltransferases; CYP450, cytochrome P450; CYB561, cytochrome b-561 domain containing protein; and $\mathrm{CaE}$, carboxylesterase.

Of all the DEGs, 68\% were annotated genes and 32\% were unknown (Figure 4B). We found that in addition to HcCht5, many other genes related to the chitin metabolism and molting hormone signal were also down-regulated in the dsHcCht5-treated group, such as chitin synthase genes ( $C H S-A$ and $C H S-B)$ and $\beta-N$-acetylglucosaminidase gene (NAG), key genes in the chitin synthesis pathway; ecdysteroid kinase gene $(E c K)$ and juvenile hormone esterase gene $(J H E)$, as well as essential genes encoding molting-related hormones (Figure 4D). These results confirmed the function of HcCht5 in chitin metabolism and the larval molting process.

Surprisingly, we also found that quite a few down-regulated DEGs were related to detoxification metabolism, including odorant-degrading enzymes (ODEs), UDP-glycosyltransferases (UGTs), cytochrome P450 (CYP450), cytochrome b-561 domain containing protein (CYB561), and carboxylesterase (CaE) (Figure 4D). Detoxification enzymes have been reported in relation to virous functions in different insect species and are often involved in insecticide, xenobiotic degradation, and pheromone metabolism. In the present study, silencing of $\mathrm{HcCht5}$ led to the down regulation of detoxification-related genes, suggesting a direct or indirect association of $\mathrm{HcCh}+5$ with detoxification metabolism during molting. To evaluate whether nonspecific dsRNAs have similar effects on immune genes, we examined all the differentially expressed immunity gene expression in untreated insects. The results showed that except for the ODE1 and CYB561 genes, the expression levels of all the other immunity genes in the untreated group were consistent with those of the dsGFP treatment. This demonstrated that the nonspecific dsRNAs do not have a similar effect on the expression of immunity-related genes (Figure S4).

\section{Discussion}

Insect chitinase genes have been widely recognized as attractive targets for the development of effective and environmentally safe insect management methods [30]. In the 
present study, we characterized the H. cunea chitinase gene (Accession number: U86877) and designated it as HcCht5. All insect chitinases belong to the GH18 family and are classified by specific structural domain organization [31]. Based on the sequence analysis, the $\mathrm{HcCh}$ t5 gene encoded a 553-amino acid protein with a signal peptide region, a catalytic domain (GH18 domain), and a chitin-binding domain (Figure 1A), possessing four conserved chitinase-catalyzed active sites (Figure 1B). These features were consistent with the characteristics of group I genes in other insect species [1,11]. Phylogenetic analysis also clustered $\mathrm{HcCht} 5$ to the group I chitinase branch (Figure S1). We therefore concluded that $\mathrm{HcCh}$ 5 5 belonged to the group I chitinase branch.

Group I chitinase (Cht5) is a well-characterized enzyme found in the integument and molting fluid $[1,30]$. We detected the expression pattern of $\mathrm{HcCht5}$ in different tissues of H. cunea larvae by RT-qPCR. The results showed that $\mathrm{HcCht5}$ was highly expressed in the epidermis, similar to the expression pattern of Cht5 in P. xylostella (PxCht5) [23] and Nilaparvata lugens (NlCht5) [32], indicating the important role of Cht5 in epidermal metabolism. We also examined the temporal expression of $\mathrm{HcCht5}$ during the different larval stages of $\mathrm{H}$. cunea. As shown in Figure 2C, the $\mathrm{HcCht5}$ transcript was only significantly highly expressed during the molting stage (L4D1 and L4D5), while its expression decreased in the inter-molting stage, which was consistent with the Northern blot results of HcCht5 (H. cunea chitinase) by Kim et al. [25] and similar to the expression pattern of Cht5s in L. migratoria (LmCht5-1 and LmCht5-2), P. xylostella (PxCht5), and C. fumiferana (CfChitinase) [6,14,23], suggesting its important roles in larval molting.

According to previous studies, most research on Cht5s in insects focused on molting; however, the function of Cht5s in metamorphosis development were slightly different between insects of different orders. In coleopteran, homopteran, and lepidopteran, Cht $5 \mathrm{~s}$ have been frequently reported to affect pupal-adult or nymph-adult molting. For example, in $T$. castaneum, a pupa-adult lethal phenotype was observed after the injection of dsRNA for Cht5, but the larva-larva and larva-pupa molts were normal [3]; in P. solenopsis, silencing $P_{s} C h t 5$ also resulted in pupation defects and failure to complete adult eclosion [24]; in S. exigua, injection dsSeCht5 (dsSeChi) at the last instar larvae demonstrated its important role during the larval-pupal and pupal-adult stages [8]. In the orthopteran insect, L. migratoria, gene duplication of chitinase 5 (Cht5-1 and Cht5-2) was identified, but only RNAi-mediated suppression of LmCht5-1 led to severe molting defects and lethality, whereas LmCht5-2 did not display any visible phenotype [14]. The distinct functions of Cht5s in insects may be related to the differences in the developmental patterns and tissue-specificity of chitinase expression. In the present study, the HcCht5 was demonstrated to be of vital importance in larva-larva molting. The injection of dsHcCht5 on the fifth day of the third larval stadium resulted in an effective RNAi response accompanied by the knockdown of HcCht5 expression and arrested molting (Figure 3, Table 1), suggesting that HcCht5 is absolutely required for the successful molting process of $H$. cunea larvae. Chitinase genes belonging to other chitinase groups have also been linked to different roles in insect development. The function of other Chts in $\mathrm{H}$. cunea will be assessed in future studies.

Importantly, we found that ds $\mathrm{HcCht5}$ injection at different larval stages resulted in different RNAi efficiencies. Treatment at the early stage of the fourth instar larvae (L4D1, L4D2 and L4D3) did not cause any phenotypic changes; however, larvae treated at the late days of the fourth instar (L4D4 and L4D5) appeared to experience abnormal molting and mortality (Table 2). In addition, a better RNAi efficiency was detected with injection at L4D5 compared with at L4D4 (Table 2). In other words, the closer to pre-molting, the better the RNAi efficiency was. The RNAi efficiency and the resulting phenotypes were variable in the target insect. These variations are reliant on several critical factors, such as the target gene transcript abundance, spatial and temporal expression profiles, and the protein stability and turnover rate of the target gene [33,34]. In H. cunea, we found that $\mathrm{HcCh}$ t5 was highly expressed during molting, while its expression decreased by about 40-60 times during inter-molting (Figure 2C), suggesting the function of HcCht5 in the molting process. When dsRNA injection occurred in L4D1, L4D2, and L4D3, premature 
intervention may have made it difficult to elicit significant RNAi phenotypes several days later, such as the injection of dsRNA targeting calreticulin and cathespsin- $L$ in Acyrthosiphon pisum, which caused a decrease in target gene expression by $35-41 \% 1-5$ days after injection, stopping at 7 days post injection [35]. Our results demonstrated that RNAi-mediated silencing of $\mathrm{HcCh}$ t5 inhibited larval molting depending on dsRNA injection time and provide a reference for the development and application of RNAi-based management of H. cunea control.

RNA-seq analysis showed that many genes related to chitin metabolism and molting hormone signaling were also down-regulated after silencing of the $\mathrm{HcCht5}$ gene. DGE analysis also found that depletion of $\mathrm{HcCht5}$ decreased the expression of many detoxification enzyme-related genes (Figure 4D). In general, insect detoxification enzymes are often involved in insecticide, xenobiotic degradation, pheromone metabolism, or odorant degradation to maintain the physiological balance within insects [36-39]. In the present study, genes related to UDP-glycosyltransferases (UGTs) and odorant-degrading enzymes (ODEs) were down-regulated after HcCht5 RNAi. UGTs catalyze the conjugation of a range of diverse small lipophilic compounds with sugars to produce glycosides, playing an important role in the detoxification of xenobiotics and in the regulation of endobiotics in insects. The expression of insect UGTs has been detected in fat bodies, the midgut, the Malpighian tubules, and even in the antennae, showing different patterns in their expression profiles and suggesting that UGT genes might have different functions $[36,40]$. Studies have found that many endogenous compounds, such as ecdysteroid hormones and cuticle tanning precursors, are glycosylated by UGT enzymes [36,41]. Recently, a putative UGT from Heterorhabditis bacteriophora (Hb-ugt-1) was examined, and its activity was found to likely involve the inactivation of ecdysone [42]. ODEs belong to olfactory proteins, playing crucial roles in the responses triggered by external chemical stimuli [43]. Insect ODEs include multiple enzyme families typically expressed in the sensillar lymph and likely involved in the fast inactivation of odorants to maintain the sensitivity of the olfactory system [44]. Few studies have focused on the effect of insect chitinase on detoxification metabolism, although detoxification enzymes are functionally diversified in insects. However, detoxification was considered indispensable for ecdysis. During insect molting, the molting fluid exudes multiple proteins for the recycling of old cuticles, and also produces a great deal of toxic molecules [45]. Successful ecdysis requires all molting proteins to work together, including in detoxification $[46,47]$. Therefore, we speculated that the down regulation of detoxification-related genes after $\mathrm{HcCht5}$ RNAi might be due to the inability to molt and the disruption of the insect physiological balance. Further studies are required to confirm this hypothesis.

\section{Conclusions}

Overall, we characterized the HcCht5 gene in H. cunea and detailed its gene function in the larval molting process through RNAi and RNA-seq. Our current findings demonstrated that the RNAi-mediated silencing of $\mathrm{HcCht5}$ arrested molting and caused larval mortality depending on the dsRNA injection time. The silencing of HcCht5 down-regulated genes related to chitin metabolism, molting hormone signaling, and detoxification metabolism.

Supplementary Materials: The following are available online at https:/ / www.mdpi.com/article / 10.3390/insects12050406/s1, Table S1: Primer sequences used in this study, Table S2: GenBank accession numbers for the phylogenetic tree used in this study, Table S3: Expression stability values of the reference genes for different developmental stages and different tissues, Table S4: Summary of the RNA sequencing data, Table S5: DEGs between the dsGFP and dsHcCht5 samples, Figure S1: Phylogenetic tree of chitinases from different insect species, Figure S2: Correlation analysis of RNA-seq samples, Figure S3: RT-qPCR validation of 10 selected RNA-seq-based DGEs, Figure S4: The expression levels of the differentially expressed immunity genes in the untreated group, dsGFP-treated group, and dsHcCht5-treated group. 
Author Contributions: Z.Z. conceived and designed the study; X.Z. and F.L. reared the insects; X.Z. and Y.W. performed the experiments and analyzed the data; X.Z., Y.W., S.Z. and X.K. wrote and reviewed the manuscript. All authors have read and agreed to the published version of the manuscript.

Funding: This research was funded by the National Key Research and Development Program of China (2018YFD0600203), the Fundamental Research Funds of CAF (CAFYBB2020QC001), and the Special Fund for Forest Scientific Research in the Public Welfare (201504302).

Institutional Review Board Statement: Not applicable.

Data Availability Statement: The data presented in this study are available in article.

Conflicts of Interest: The authors declare no conflict of interest.

\section{References}

1. Arakane, Y.; Muthukrishnan, S. Insect chitinase and chitinase-like proteins. Cell. Mol. Life Sci. 2010, 67, 201-216. [CrossRef]

2. Zhu, K.Y.; Merzendorfer, H.; Zhang, W.; Zhang, J.; Muthukrishnan, S. Biosynthesis, turnover, and functions of chitin in insects. Annu. Rev. Entomol. 2016, 61, 177-196. [CrossRef]

3. Zhu, Q.; Arakane, Y.; Beeman, R.W.; Kramer, K.J.; Muthukrishnan, S. Functional specialization among insect chitinase family genes revealed by RNA interference. Proc. Natl. Acad. Sci. USA 2008, 105, 6650-6655. [CrossRef]

4. Kramer, K.J.; Corpuz, L.; Choi, H.K.; Muthukrishnan, S. Sequence of a cDNA and expression of the gene encoding epidermal and gut chitinases of Manduca-sexta. Insect Biochem. Mol. 1993, 23, 691-701. [CrossRef]

5. Shinoda, T.; Kobayashi, J.; Matsui, M.; Chinzei, Y. Cloning and functional expression of a chitinase cDNA from the common cutworm, Spodoptera litura, using a recombinant baculovirus lacking the virus-encoded chitinase gene. Insect Biochem. Mol. 2001, 31, 521-532. [CrossRef]

6. Zheng, Y.; Zheng, S.; Cheng, X.; Ladd, T.; Lingohr, E.J.; Krell, P.J.; Arif, B.M.; Retnakaran, A.; Feng, Q. A molt-associated chitinase cDNA from the spruce budworm, Choristoneura fumiferana. Insect Biochem. Mol. 2002, 32, 1813-1823. [CrossRef]

7. Ahmad, T.; Rajagopal, R.; Bhatnagar, R.K. Molecular characterization of chitinase from polyphagous pest Helicoverpa armigera. Biochem. Biophys. Res. Commun. 2003, 310, 188-195. [CrossRef] [PubMed]

8. Zhang, D.; Chen, J.; Yao, Q.; Pan, Z.; Chen, J.; Zhang, W. Functional analysis of two chitinase genes during the pupation and eclosion stages of the beet armyworm Spodoptera exigua by RNA interference. Arch. Insect Biochem. 2012, 79, 220-234. [CrossRef]

9. Li, Y.L.; Song, H.F.; Zhang, X.Y.; Li, D.Q.; Zhang, T.T.; Ma, E.B.; Zhang, J.Z. Heterologous expression and characterization of two chitinase 5 enzymes from the migratory locust Locusta migratoria. Insect Sci. 2016, 23, 406-416. [CrossRef] [PubMed]

10. Yang, X.; Zhou, C.; Long, G.; Yang, H.; Chen, C.; Jin, D. Characterization and functional analysis of chitinase family genes involved in nymph-adult transition of Sogatella furcifera. Insect Sci. 2020, 1-16. [CrossRef]

11. Tetreau, G.; Cao, X.; Chen, Y.R.; Muthukrishnan, S.; Jiang, H.; Blissard, G.W.; Kanost, M.R.; Wang, P. Overview of chitin metabolism enzymes in Manduca sexta: Identification, domain organization, phylogenetic analysis and gene expression. Insect Biochem. Mol. 2015, 62, 114-126. [CrossRef]

12. Zhang, J.; Zhang, X.; Arakane, Y.; Muthukrishnan, S.; Kramer, K.J.; Ma, E.; Zhu, K.Y. Comparative genomic analysis of chitinase and chitinase-like genes in the African malaria mosquito (Anopheles gambiae). PLoS ONE 2011, 6, e19899. [CrossRef]

13. Zhu, Q.; Arakane, Y.; Beeman, R.W.; Kramer, K.J.; Muthukrishnan, S. Characterization of recombinant chitinase-like proteins of Drosophila melanogaster and Tribolium castaneum. Insect Biochem. Mol. 2008, 38, 467-477. [CrossRef]

14. Li, D.; Zhang, J.; Wang, Y.; Liu, X.; Ma, E.; Sun, Y.; Li, S.; Zhu, K.Y.; Zhang, J. Two chitinase 5 genes from Locusta migratoria: Molecular characteristics and functional differentiation. Insect Biochem. Mol. 2015, 58, 46-54. [CrossRef] [PubMed]

15. Ge, X.; He, S.; Zhu, C.; Wang, T.; Xu, Z.; Zong, S. Projecting the current and future potential global distribution of Hyphantria cunea (Lepidoptera: Arctiidae) using CLIMEX. Pest. Manag. Sci. 2019, 75, 160-169. [CrossRef] [PubMed]

16. Zhu, H.; Shen, Y.; Cui, J.; Wang, A.; Li, N.; Wang, C.; Cui, B.; Sun, C.; Zhao, X.; Wang, C.; et al. Avermectin loaded carboxymethyl cellulose nanoparticles with stimuli-responsive and controlled release properties. Ind. Crop. Prod. 2020, 152, 112497. [CrossRef]

17. Yang, Z.Q.; Wei, J.R.; Wang, X.Y. Mass rearing and augmentative releases of the native parasitoid Chouioia cunea for biological control of the introduced fall webworm Hyphantria cunea in China. BioControl 2006, 51, 401-418. [CrossRef]

18. Su, M.; Fang, Y.; Tao, W.; Yan, G.; Ma Wan, E.; Zhang, Z. Identification and field evaluation of the sex pheromone of an invasive pest, the fall webworm Hyphantria cunea in China. Chin. Sci. Bull. 2008, 53, 555-560. [CrossRef]

19. Zhang, L.W.; Kang, K.; Liu, Y.J.; Zhang, J.; Sun, L.; Zhan, C.; Huang, C.C.; Jiang, L.Y.; Ye, K.Y.; Ding, D.G. Evaluation of Beauveria bassiana isolates as potential agents for control of Hyphantria cunea (Lepidoptera Arctiidae). Acta Entomol. Sin. 2016, 59, 111-118.

20. Deng, Y.; Li, F.; Rieske, L.K.; Sun, L.L.; Sun, S.H. Transcriptome sequencing for identification of diapause-associated genes in fall webworm, Hyphantria cunea Drury. Gene 2018, 668, 229-236. [CrossRef]

21. Wu, N.; Zhang, S.; Li, X.; Cao, Y.; Liu, X.; Wang, Q.; Liu, Q.; Liu, H.; Hu, X.; Zhou, X.J.; et al. Fall webworm genomes yield insights into rapid adaptation of invasive species. Nat. Ecol. Evol. 2019, 3, 105-115. [CrossRef] [PubMed]

22. Chen, Q.; Zhao, H.; Wen, M.; Li, J.; Zhou, H.; Wang, J.; Zhou, Y.; Liu, Y.; Du, L.; Kang, H.; et al. Genome of the webworm Hyphantria cunea unveils genetic adaptations supporting its rapid invasion and spread. BMC Genom. 2020, 21, 242. [CrossRef] 
23. Zhu, B.; Shan, J.; Li, R.; Liang, P.; Gao, X. Identification and RNAi-based function analysis of chitinase family genes in diamondback moth, Plutella xylostella. Pest. Manag. Sci. 2019, 75, 1951-1961. [CrossRef]

24. Omar, M.A.A.; Ao, Y.; Li, M.; He, K.; Xu, L.; Tong, H.; Jiang, M.; Li, F. The functional difference of eight chitinase genes between male and female of the cotton mealybug, Phenacoccus solenopsis. Insect Mol. Biol. 2019, 28, 550-567. [CrossRef] [PubMed]

25. Kim, M.G.; Shin, S.W.; Bae, K.S.; Kim, S.C.; Park, H.Y. Molecular cloning of chitinase cDNAs from the silkworm, Bombyx mori and the fall webworm, Hyphantria cunea. Insect Biochem. Mol. 1998, 28, 163-171. [CrossRef]

26. Chenna, R.; Sugawara, H.; Koike, T.; Lopez, R.; Gibson, T.J.; Higgins, D.G.; Thompson, J.D. Multiple sequence alignment with the Clustal series of programs. Nucleic Acids Res. 2003, 31, 3497-3500. [CrossRef]

27. Zhang, J.Z.; Zhang, X.; Arakane, Y.; Muthukrishnan, S.; Kramer, K.J.; Ma, E.; Zhu, K.Y. Identification and characterization of a novel chitinase-like gene cluster $(A g C h+5)$ possibly derived from tandem duplications in the African malaria mosquito, Anopheles gambiae. Insect Biochem. Mol. 2011, 41, 521-528. [CrossRef]

28. Grabherr, M.G.; Haas, B.J.; Yassour, M.; Levin, J.Z.; Thompson, D.A.; Amit, I.; Adiconis, X.; Fan, L.; Raychowdhury, R.; Zeng, Q.; et al. Full-length transcriptome assembly from RNA-Seq data without a reference genome. Nat. Biotechnol. 2011, 29, 644-652. [CrossRef]

29. Li, B.; Dewey, C.N. RSEM: Accurate transcript quantification from RNA-Seq data with or without a reference genome. BMC Bioinform. 2011, 12, 323. [CrossRef]

30. Kramer, K.J.; Muthukrishnan, S. Insect chitinases: Molecular biology and potential use as biopesticides. Insect Biochem. Mol. 1997, 27, 887-900. [CrossRef]

31. Funkhouser, J.D.; Aronson, N.N., Jr. Chitinase family GH18: Evolutionary insights from the genomic history of a diverse protein family. BMC Evol. Biol. 2007, 7, 96. [CrossRef]

32. Xi, Y.; Pan, P.L.; Ye, Y.X.; Yu, B.; Xu, H.J.; Zhang, C.X. Chitinase-like gene family in the brown planthopper, Nilaparvata lugens. Insect Mol. Biol. 2015, 24, 29-40. [CrossRef]

33. Scott, J.G.; Michel, K.; Bartholomay, L.C.; Siegfried, B.D.; Hunter, W.B.; Smagghe, G.; Zhu, K.Y.; Douglas, A.E. Towards the elements of successful insect RNAi. J. Insect Physiol. 2013, 59, 1212-1221. [CrossRef] [PubMed]

34. Jain, R.G.; Robinson, K.E.; Fletcher, S.J.; Mitter, N. RNAi-Based functional genomics in hemiptera. Insects 2020, 11, 557. [CrossRef]

35. Jaubert-Possamai, S.; Le Trionnaire, G.; Bonhomme, J.; Christophides, G.K.; Rispe, C.; Tagu, D. Gene knockdown by RNAi in the pea aphid Acyrthosiphon pisum. BMC Biotechnol. 2007, 7, 63. [CrossRef] [PubMed]

36. Ahn, S.J.; Vogel, H.; Heckel, D.G. Comparative analysis of the UDP-glycosyltransferase multigene family in insects. Insect Biochem. Mol. 2012, 42, 133-147. [CrossRef] [PubMed]

37. Li, X.; Schuler, M.A.; Berenbaum, M.R. Molecular mechanisms of metabolic resistance to synthetic and natural xenobiotics. Insect Biochem. Mol. 2007, 52, 231-253. [CrossRef] [PubMed]

38. Wojtasek, H.; Leal, W.S. Degradation of an alkaloid pheromone from the pale-brown chafer, Phyllopertha diversa (Coleoptera: Scarabaeidae), by an insect olfactory cytochrome P450. FEBS Lett. 1999, 458, 333-336. [CrossRef]

39. Zhang, Y.X.; Wang, W.L.; Li, M.Y.; Li, S.G.; Liu, S. Identification of putative carboxylesterase and aldehyde oxidase genes from the antennae of the rice leaffolder, Cnaphalocrocis medinalis (Lepidoptera: Pyralidae). J. Asia-Pac. Entomol. 2017, 20, 907-913. [CrossRef]

40. Huang, F.F.; Chai, C.L.; Zhang, Z.; Liu, Z.H.; Dai, F.Y.; Lu, C.; Xiang, Z.H. The UDP-glucosyltransferase multigene family in Bombyx mori. BMC Genom. 2008, 9, 563. [CrossRef]

41. Hopkins, T.L.; Kramer, K.J. Insect cuticle sclerotization. Annu. Rev. Entomol. 1992, 37, 273-302. [CrossRef]

42. Kenney, E.; Yaparla, A.; Hawdon, J.M.; O’Halloran, D.M.; Grayfer, L.; Eleftherianos, I. A putative UDP-glycosyltransferase from Heterorhabditis bacteriophora suppresses antimicrobial peptide gene expression and factors related to ecdysone signaling. Sci. Rep. 2020, 10. [CrossRef] [PubMed]

43. Leal, W.S. Odorant reception in insects: Roles of receptors, binding proteins, and degrading enzymes. Annu. Rev. Entomol. 2013, 58, 373-391. [CrossRef]

44. He, P.; Durand, N.; Dong, S.L. Editorial: Insect olfactory proteins (from gene identification to functional characterization). Front. Physiol. 2019, 10, 1313. [CrossRef] [PubMed]

45. Zhang, J.; Lu, A.; Kong, L.; Zhang, Q.; Ling, E. Functional analysis of insect molting fluid proteins on the protection and regulation of ecdysis. J. Asia-Pac. Entomol. 2014, 289, 35891-35906. [CrossRef] [PubMed]

46. Yu, Q.Y.; Lu, C.; Li, W.L.; Xiang, Z.H.; Zhang, Z. Annotation and expression of carboxylesterases in the silkworm, Bombyx mori. BMC Genom. 2009, 10, 553. [CrossRef] [PubMed]

47. Barski, O.A.; Tipparaju, S.M.; Bhatnagar, A. The aldo-keto reductase superfamily and its role in drug metabolism and detoxification. Drug Metab. Rev. 2008, 40, 553-624. [CrossRef] [PubMed] 